\section{A Conducting Cylinder for Modeling Human Body Presence in Indoor Propagation Channel}

\author{
Mohamad Ghaddar, Larbi Talbi, Tayeb A. Denidni, and \\ Abderazik Sebak
}

\begin{abstract}
We demonstrate that in indoor radio propagation modeling, the presence of the human body may be approximated by a conducting circular cylinder at microwave frequencies. Therefore, a perfect tool such as the uniform theory of diffraction may be used to predict the diffracted field over a smooth circular surface. To validate the model, vertically and horizontally polarized continuous wave $(\mathrm{CW})$ measurements were performed at $10.5 \mathrm{GHz}$ between two fixed terminals inside a room along with the presence of an obstacle (person or metallic cylinder) moving along predetermined parallel and perpendicularly crossing paths with respect to the line-of-sight direction. Results indicate that there is a strong correlation between the effects of the human body and those of a conducting circular cylinder. The simulation results successfully agree with the $\mathrm{CW}$ experimental measurements.
\end{abstract}

Index Terms-Human body modeling, indoor radio propagation, obstruction, polarization, uniform theory of diffraction (UTD).

\section{INTRODUCTION}

The availability of high frequency bands has made wireless systems an attractive option for high-speed digital communications such as wireless local area networks (WLAN) [1]. The increasing use of wireless systems in indoor applications requires a greater knowledge of the indoor propagation environment especially when they are subject to complex propagation phenomena, with the presence of moving people. Moreover, at microwave frequencies and above, the human body constitutes a practically insurmountable reflector or an obstacle for electromagnetic waves [2]. Thus, it is of interest to assess the impact of the presence of moving people presence on the characteristics of the propagation channel, and consequently, on the transceiver design of indoor radio systems. This implies realistic system performance predictions requiring accurate modeling of these effects under various conditions. Several empirical [3] and statistical [4] models for the human body shadowing are available in the literature. However, there is no deterministic published model introduced to simulate the effects of the human body on an indoor environment. Indeed, the measurements and simulated data presented in publications often imply a static radio environment, where there are no external disturbances on the radio channel.

For a comprehensive approximation of the human body a special interest has been focused on its shape and material, while assuming it can be modeled by a circular cylinder. The uniform theory of diffraction (UTD) may be used with the elliptic or the circular cylinder shape, since the elliptic cylinder is a special case of the circular cylinder. Both shapes yield results that approximate the measurements. To analyze the effects of the human body on the received signal, a set of measurements was conducted at $10.5 \mathrm{GHz}$, and has established a strong correlation

Manuscript received March 2, 2005; revised March 6, 2007. This work was supported by the Natural Sciences and Engineering Research Council of Canada (NSERC).

M. Ghaddar and T. A. Denidni are with the INRS-EMT, University of Quebec, Montreal, QC H5A 1K6, Canada.

L. Talbi is with the Computer Sciences and Engineering Department, University of Quebec in Outaouais, QC J8X 3X7, Canada (e-mail: talbi@uqo.ca).

A. Sebak is with the Electrical and Computer Engineering Department, Concordia University, Montreal, QC H3G 1M8, Canada.

Color versions of one or more of the figures in this paper are available online at http://ieeexplore.ieee.org.

Digital Object Identifier 10.1109/TAP.2007.908563

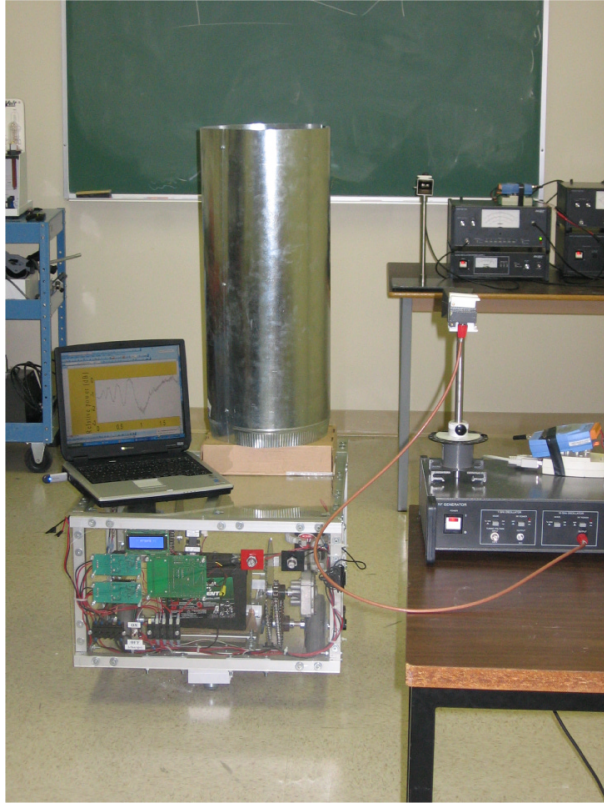

Fig. 1. Photography of the indoor propagation measurement setup.

between the effects of a conducting cylinder and those of the human body. Therefore, in this paper, the human body is approximated by a perfectly conducting circular cylinder. A series of measurements and simulations were performed to provide a mean to investigate the human body scattering and shadowing effects.

\section{Measurement Setup AND Scenarios}

Continuous wave $(\mathrm{CW})$ experimental measurements were carried out using a transmitter generating a pure sine wave at a power level of $3 \mathrm{dBm}$ at a frequency of $10.5 \mathrm{GHz}$ via vertically and horizontally polarized horn antennas with a gain of $14.5 \mathrm{~dB}$ and beamwidths of $29^{\circ}$ and $38^{\circ}$ in the $\mathrm{E}$ and $\mathrm{H}$ planes, respectively. At the receiving side, the same type of antenna was used along with a Gunn type oscillator to downconvert the received signal to $100 \mathrm{MHz}$. After amplification, the signal was recorded using an analog/digital converter (card PCI 6014). A PC involving Labview software was used to perform signal monitoring and data acquisition. Both transmitting and receiving antennas were adjusted to a height of $1.65 \mathrm{~m}$ and were separated by a distance of $3.50 \mathrm{~m}$ to obtain the best line-of-sight (LOS) signal level. One should note that reflections from the walls and the ground are negligible, as compared to the LOS and the scattering off the obstacle. The room, where the measurements took place, was emptied, except for instruments, to allow the free movement of one person. Only one person was allowed to move during the experiments. Walking was done at a continuous low speed of approximately $25 \mathrm{~cm} / \mathrm{s}$ along predetermined parallel and perpendicularly crossing paths with respect to the LOS direction. Another series of measurements was performed for each of the above cases, where the human body was then replaced by a conducting circular cylinder (radius $=20 \mathrm{~cm}$, length $=95 \mathrm{~cm}$ ), mounted on a fully automated trolley that also moved at the same speed. The cylinder was lifted so that its upper edge remained at a height of $2.50 \mathrm{~m}$ above ground, as shown in Fig. 1.

In order to validate the proposed human body model in the indoor propagation channel, two different scenarios were considered. They consisted in the blocking of the LOS path or walking beside the LOS path between the transmitter and the receiver, as illustrated in Fig. 2. 


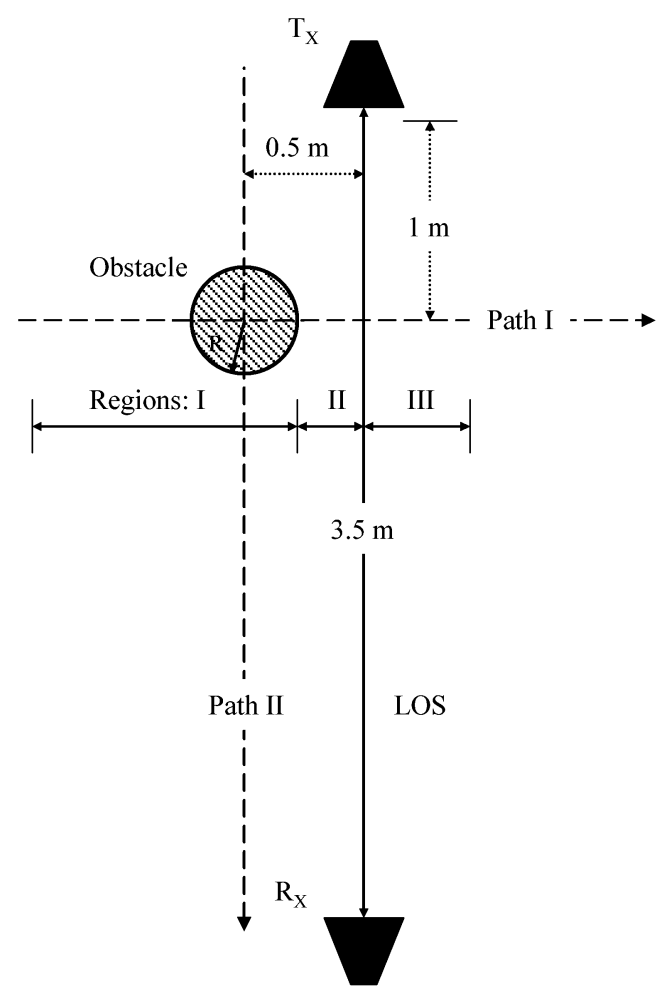

Fig. 2. Top view of obstacle crossing LOS (Path I) or moving on the side of LOS (Path II).

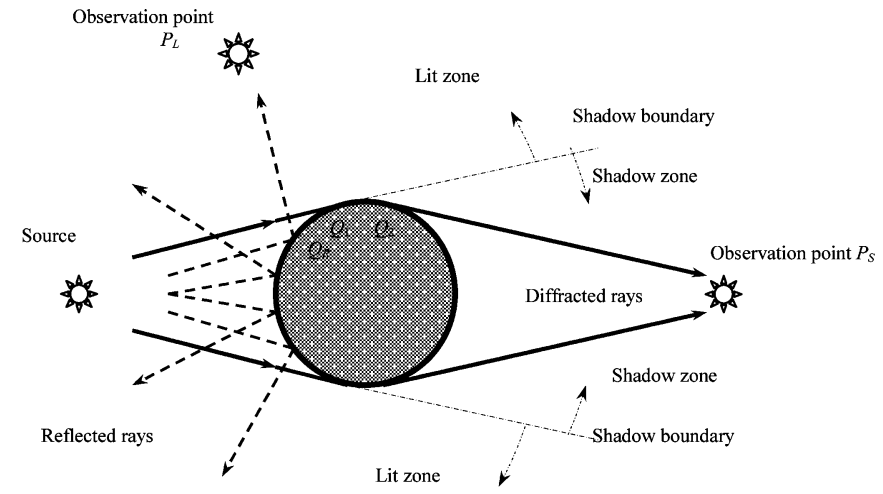

Fig. 3. Geometrical configuration of the diffraction over a smooth convex surface.

While maintaining both the transmitter and the receiver antennas at fixed positions, we investigated the behavior of propagation due to the movement of an obstacle (human versus cylinder). In the first instance, the obstacle was crossing the LOS path (Path I) at a right angle. In the second instance, the obstacle was moving along a path that was parallel to the LOS path (Path II).

\section{MODELING CONSIDERATIONS}

In this section, a practical model which deterministically estimates the human body scattering effects is investigated. The details of the theoretical analysis can be found in [5] and for the purpose of this work only the main equations used in the simulation are given below. The geometrical configuration of the diffraction problem being investigated is illustrated in Fig. 3. Referring to this figure, one notes that an extension of the incident ray beyond the grazing point at $\mathrm{Q}_{1}$ on the convex surface of the cylinder defines the shadow boundary (SB), which divides the space exterior to the surface into the lit and shadow zones. Regions on either side of the SB are the SB transition zones in which the field description changes rapidly and continuously from the geometrical optics (GO) field to the entirely surface diffracted field. The form of the UTD solution for the total electric field for $\mathrm{P}_{\mathrm{L}}$ in the lit zone may be expressed as

$$
\vec{E}\left(P_{L}\right) \approx \vec{E}^{i}\left(P_{L}\right)+\vec{E}^{i}\left(Q_{R}\right) \cdot \overline{\bar{R}} \cdot A_{L}(s) e^{-j k s^{r}}
$$

where $\vec{E}^{i}\left(Q_{R}\right)$ is the E-field incident on the cylinder at $Q_{R}$ as illustrated in Fig. 3 and where $\vec{E}^{i}\left(P_{L}\right)$ is the direct E-field incident on the observation point. $A_{L}(s)$ is the divergence factor (or spreading factor) associated with the reflected field. This governs the amplitude variation of the GO field along the ray path. However, the spreading factor is a function of the principal radii of curvature of the reflected wavefront at $Q_{R}$ and the distance along the reflected ray. This distance is computed from the point of reflection on the surface of the obstacle to the observation point $P_{L} . \overline{\bar{R}}$ is the dyadic reflection coefficient. The form of the UTD solution for the total electric field for $P_{s}$ in the shadow zone may be expressed as

$$
\vec{E}(P s) \approx \vec{E}^{i}\left(Q_{1}\right) \cdot \overline{\bar{T}} \cdot A_{s}(s) \cdot e^{-j k s^{d}}
$$

where $\vec{E}^{i}\left(Q_{1}\right)$ is the electric field incident on the cylinder at the grazing point $Q_{1}$ as observed from Fig. 3, $A_{s}(s)$ is the divergence factor (or spreading factor) associated with the diffracted field, $\overline{\bar{T}}$ is the dyadic transfer function which relates the diffracted field from $Q_{2}$ to the field incident at $Q_{1}$.

\section{MEASUREMENTS AND VALIDATION}

\section{A. Obstacle Crossing the LOS Path}

A series of CW measurements were conducted for an obstacle moving along Path $\mathrm{I}$ at $1 \mathrm{~m}$ away from the transmitting antenna as described previously. The measured results for this scenario are shown in Fig. 4, where the total received power signal is recorded as a function of the relative position of the mobile obstacle on the planned path with respect to the transmitting and receiving antenna positions. In fact, in the case where the obstacle was a person, the measurements are given on Fig. 4(a). For the case where the cylinder was the obstacle, the measurements are shown on Fig. 4(b). For comparison purposes, all the data were normalized to the maximum received power. It is worth noting that at $10.5 \mathrm{GHz}$ there was no significant power absorption observed by the human body. A decay of less than $1 \mathrm{~dB}$ was experienced. Fig. 4-I displays two signals with relative power signal variations ranging from $-26 \mathrm{~dB}$ to $0 \mathrm{~dB}$, in the case of a vertically polarized electric field. However, these signals range from $-18.5 \mathrm{~dB}$ to $0 \mathrm{~dB}$ in the case of the horizontal polarization, as shown in Fig. 4-II. Although the propagation losses increase significantly while an obstacle blocks the LOS path with either polarization, the horizontal polarization corresponds nevertheless to a lower loss.

In terms of quality, the obtained results clearly display an interesting correspondence between the measurements involving a human body and the measurements involving a metallic cylinder. The measured signal variations associated with the cylinder or the human body compare favorably, in terms of fading, periodicity and power signal decay. Measured signals resulting from the occultation by a human body or by a metallic cylinder exhibit a propagation loss of $25 \mathrm{~dB}$ in the worst channel conditions, i.e., when the diffracted field is at its lowest value. Geometrically this occurs when the obstacle is almost entirely blocking the channel link. Minor differences may be associated with the fact that human movement is not as uniform as one would expect since its 
(a)

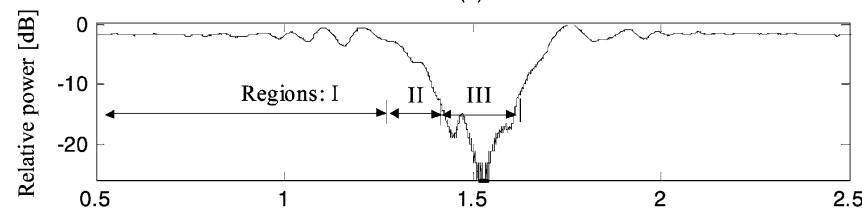

(b)

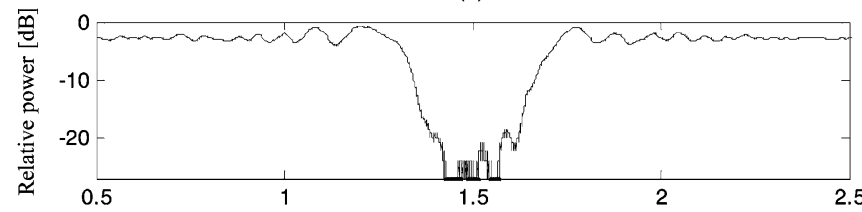

(c)

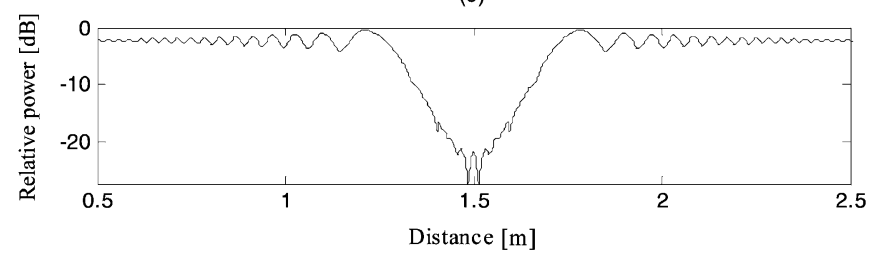

I.

(a)

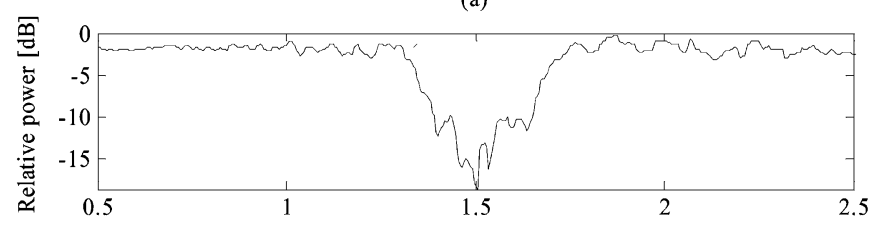

(b)

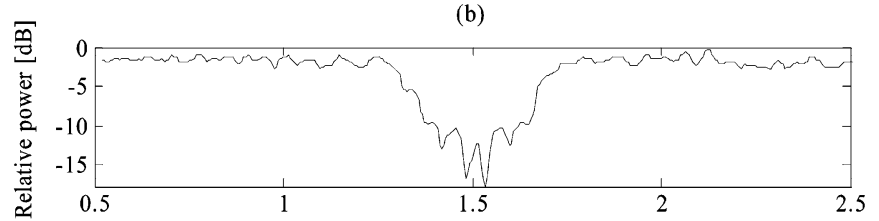

(c)

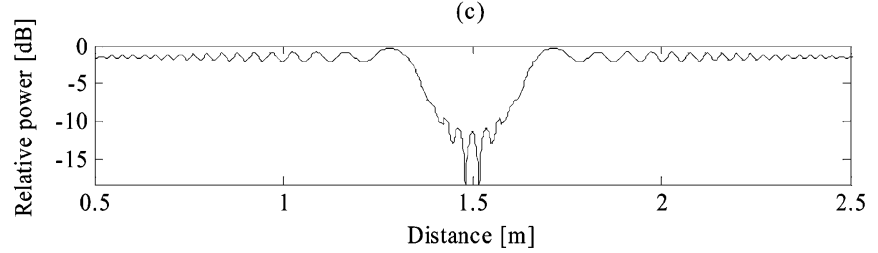

II.

Fig. 4. Measured and simulated results obtained at $10.5 \mathrm{GHz}$ for obstacle moving along path I: (a) Experimental results for human body, (b) experimental results for metallic cylinder, (c) simulated results for perfectly conducting cylinder. I. Vertical polarization and II. horizontal polarization.

speed is variable and the motion of the arms may also affect the received signal intensity. Moreover, at microwave frequencies or higher clothes may have an effect on the signal propagation, since the surface roughness of the clothes which is of the order of a tenth of a wavelength. Besides, the light weight and the height of the empty circular cylinder may be the source of some vibrations during its trip on the mobile trolley. Therefore, its movement was not as smooth as desired.

To relate the obstacle movement to the received signal envelope, it is necessary to take into consideration the nature and mechanism of propagation during this movement. Therefore, three regions along the path of the obstacle are defined (see Fig. 2).

a) Region I: When the obstacle travels toward the $\operatorname{LOS}(0.5-1.3 \mathrm{~m})$, the receiver tends to be in the deep lit region of the illuminating field, where the total received signal is expected to be induced by the interaction between the reflected (or diffracted) ray from the obstacle and the LOS component. b) Region II: However, when the obstacle appears in the vicinity of the LOS (1.3-1.4 m), on either side of the shadow boundary, which is located between the lit and shadow zones, known as the transition region, the reflected electromagnetic field amplifies to changes continuously and smoothly from the geometrical optics reflected field to the surface diffracted field, or vice versa.

c) Region III: As the obstacle blocks the LOS, the receiver appears to be in the deep shadow region (1.4-1.7 $\mathrm{m}$ ) of the illuminating field, consequently, the total received signal envelope might be induced entirely by the obstacle diffracted field.

The UTD was used in the above three regions under the assumption of a perfectly conducting cylinder with a radius of $20 \mathrm{~cm}$ and considering the above three regions. The simulation results are given in Fig. 4(c) and reveal an excellent agreement with the measurements.

\section{B. Obstacle Moving Parallel to the LOS Path}

In this scenario, an obstacle traveling at a constant low velocity moves along a parallel path to the LOS path at a distance of 0.5 $\mathrm{m}$. The layout is depicted in Fig. 2 (Path II). Since the obstacle is located in the deep lit zone of the electromagnetic field, the received power signal is likely to be induced by the interaction between the ray reflected from the obstacle and the unobstructed LOS component. The measurement results for the human body and the cylinder are given respectively in Fig. 5(a) and (b), and show an interesting agreement in terms of signal amplitude variations within a range of $3 \mathrm{~dB}$. The minor differences between the signals may be attributed to the same reasons discussed in the previous scenario. It is worth mentioning that the low rate of signal amplitude variation mainly occurs in the middle region, between the transmitter and the receiver, since this region exhibits a low rate of phase variations caused by the differential path between the reflected ray and the LOS ray. Simulation results for this scenario are provided in Fig. 5(c) and demonstrate also a fair agreement with the experimental results.

Interesting observations regarding the polarization may be made. For the vertical polarization, the amplitude variations of the received signal fall within a range of $3 \mathrm{~dB}$ as shown in Fig. 5-I. However, Fig. 5-II reveals that the received signal envelope falls within a range of $2 \mathrm{~dB}$ for the horizontal polarization. Hence, the radio channel, when operating with the horizontal polarization, leads to a reduction of the propagation loss by $1 \mathrm{~dB}$. Both polarizations agree with the theoretical predictions. The signal variations corresponding to each polarization appear to be $180^{\circ}$ out of phase with respect to each other, i.e., while the signal resulting from the vertical polarization reaches its maximum the horizontally polarized signal reaches its minimum and vice versa.

It is of interest to characterize the radio link in the presence of human disturbances. Therefore, it is useful to observe the statistical distributions of various data. The cumulative distribution functions (CDFs) of the received signals corresponding to human displacements along Path I and Path II are given in Figs. 6 and 7, respectively. During the crossing path, as long as the obstacle is still in Region I (lit zone), i.e., unobstructed LOS between the transmitting and receiving antennas, the channel fading tends to behave as a Ricean channel with $\mathrm{K}=19 \mathrm{~dB}$; $K$-factor is defined as the ratio of signal power in dominant component over the scattered power, as shown in Fig. 6. Due to the appearance of the obstacle in Region III (shadowing zone), blocking the LOS after leaving the transition zone (Region II), the channel experiences a serious degradation and behaves as Rayleigh channel. Under these circumstances, the horizontally polarized antennas provide better channel performance in terms of loss by approximately $5 \mathrm{~dB}$.

During displacements along Path II a Ricean distribution of intensities is expected since the channel presents an unobstructed line-of-sight between the transmit and receive antennas. More precisely, in Path II, each polarization leads to a different channel behavior, as shown in 
(a)

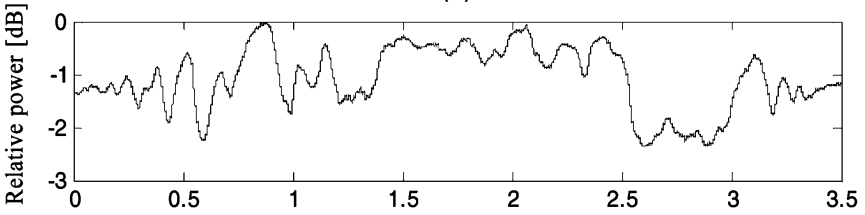

(b)

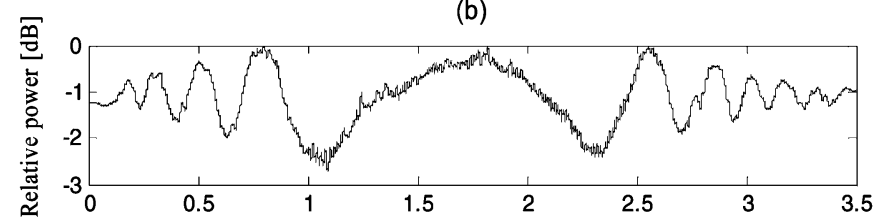

(c)

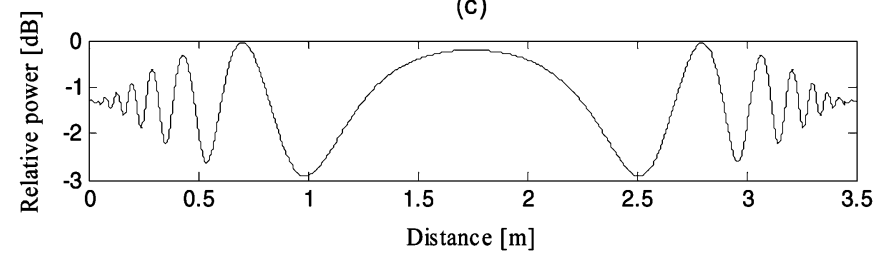

I.

(a)

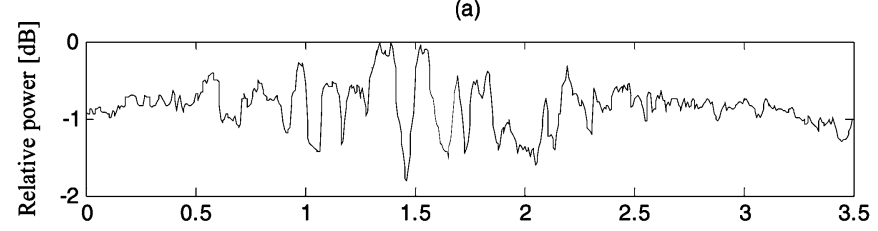

(b)

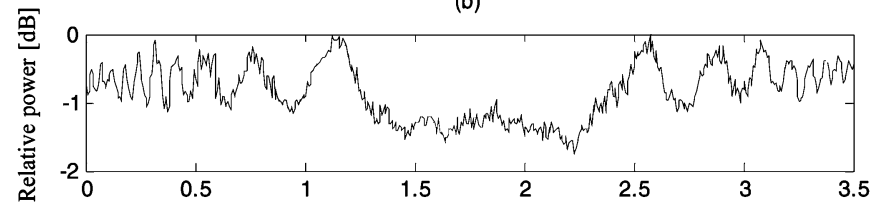

(c)

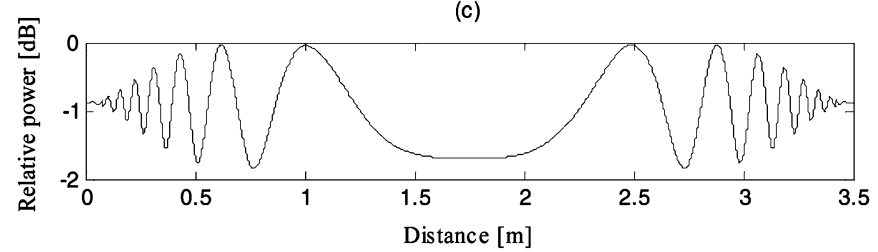

II.

Fig. 5. Measured and simulated results obtained at $10.5 \mathrm{GHz}$ for obstacle moving along path II: (a) Experimental results for human body, (b) experimental results for metallic cylinder, (c) simulated results for perfectly conducting cylinder. I. Vertical polarization and II. horizontal polarization.

Fig. 7. In fact, when the antennas are vertically polarized, the received signal distribution approximates a Ricean CDF with $K=14 \mathrm{~dB}$. However, for the horizontal polarization, the Rice factor of the channel is $K=20 \mathrm{~dB}$, which means that the horizontal polarization mitigates the effects of the rays reflected from the human body.

\section{CONCLUSION}

This paper demonstrates that a conducting circular cylinder can be used to model the presence of the human body in the indoor propagation channel. Such a shape, easily treated with the UTD, can be incorporated in the ray-tracing simulation technique to deal with the human disturbances of the propagation channel encountered at microwave frequencies and beyond. To experimentally validate the model, two scenarios were studied: crossing the LOS path and moving parallel to the LOS path while using a $10.5 \mathrm{GHz}$ radio propagation channel between two fixed terminals. The various results obtained in this investigation

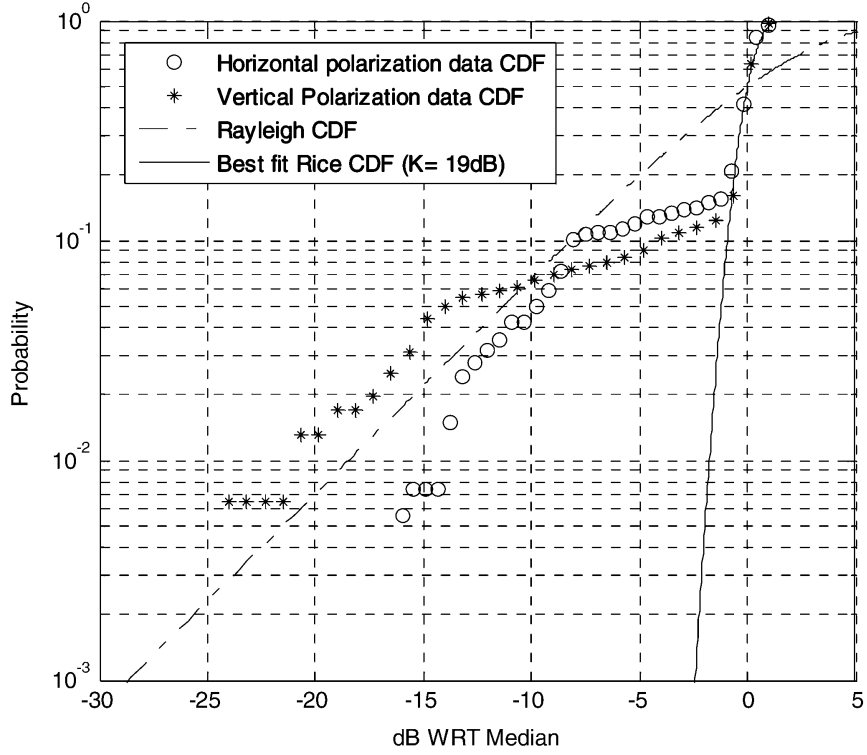

Fig. 6. Experimental data CDF of human moving along Path I.

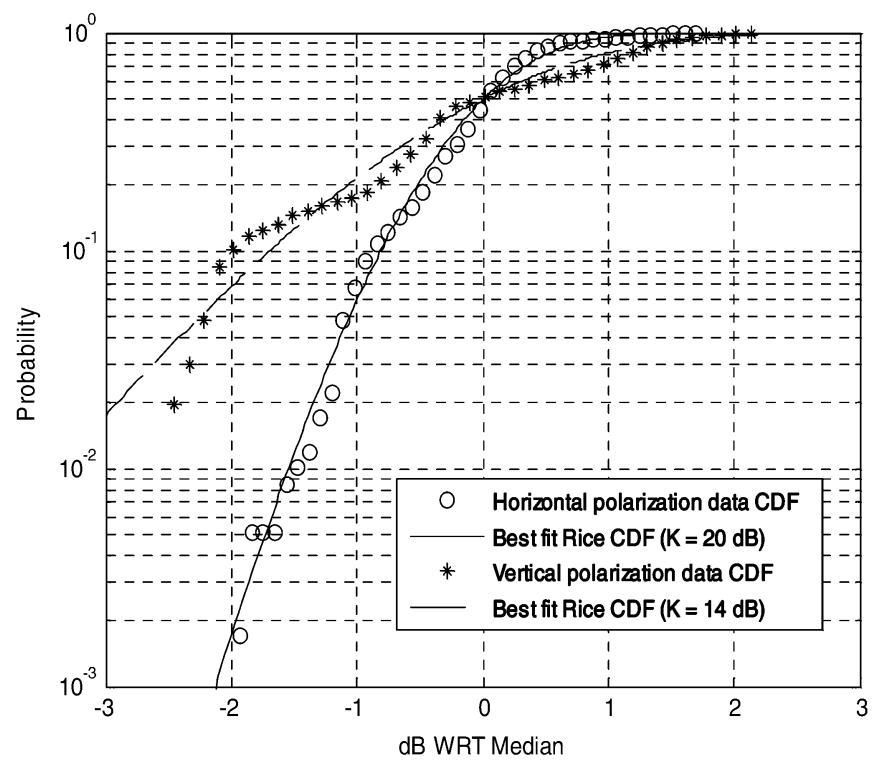

Fig. 7. Experimental data CDF of human moving along Path II.

show a strong correlation between a circular conducting cylinder and the human body. They also show a strong dependence of the received power on the signal polarization. The CDFs of the measurements indicate that horizontally polarized antennas can offer a relatively higher performance for the environment under consideration.

\section{ACKNOWLEDGMENT}

The authors gratefully acknowledge the anonymous reviewers for their comments toward the improvement of this paper.

\section{REFERENCES}

[1] T. S. Rappaport, A. Annamalai, R. M. Buehrer, and W. H. Traniter, "Wireless communications: Past events and future perspective," IEEE Commun. Mag., pp. 148-161, May 2002.

[2] L. Talbi, "Human disturbance of indoor EHF wireless channel," Electron. Lett., vol. 37, no. 22, pp. 1361-1363, Oct. 2001. 
[3] R. J. C. Bultitude, "Measurements, characterization and modelling of indoor 800/900 MHz radio channels for digital communications," IEEE Commun. Mag., vol. 25, pp. 5-12, June 1987.

[4] S. Obayashi and J. Zander, "A body-shadowing model for indoor radio communication environments," IEEE Trans. Antennas Propag., vol. 46, no. 6, pp. 920-927, Jun. 1998.

[5] P. H. Pathak, W. D. Burnside, and R. J. Marhefka, "A uniform GTD analysis of the diffraction of electromagnetic waves by a smooth convex surface," IEEE Trans. Antennas Propag., vol. 28, no. 5, pp. 631-642, Sep. 1980

\section{Parametric Modeling of Ultrawideband Antennas}

\author{
Y. Duroc, A. Ghiotto, T. P. Vuong, and S. Tedjini
}

\begin{abstract}
Compared to narrowband antennas, ultrawideband (UWB) antennas have particular characteristics which involve new ways to model them. This paper introduces the use of parametric modeling to efficiently model transfer functions (and associated impulse responses) of UWB antennas, and to determine an equivalent circuit of their input impedance.
\end{abstract}

Index Terms-Input impedance, parametric modeling, transfer function, transient response, ultrawideband (UWB).

\section{INTRODUCTION}

Ultrawideband (UWB) is a promising technology for future short range wireless communications with high data rates as well as radar and geolocation [1]. There are many issues involved in designing UWB systems, such as antenna design, interference, propagation, channel effects and modulation methods [2]. Especially, UWB antennas act as major pulse-shaping filters having an important impact on the receiver and the transmitter design complexities. Therefore, the performance evaluation of UWB systems should include antenna effects. With this objective, this paper introduces efficient approaches to model characteristics of UWB antennas: transfer function and antenna input impedance. Without loss of generality, a specific UWB antenna is used to give examples.

\section{SPECIFICITIES OF UWB ANTENNAS}

An antenna can be considered as a transducer transforming guided electromagnetic waves into electromagnetic waves propagating in free space, and vice versa. Standard definitions may be employed to define antenna characteristics [3]. Considering an antenna as an electromagnetic radiator, a radio frequency $(\mathrm{RF})$ engineer will be rather interested by its radiation patterns, directivity, gain, aperture, efficiency and polarization. However, considering an antenna as a circuit element, an RF circuit designer will be more interested by its input impedance, reflection coefficient and voltage standing wave ratio. Generally, when considering narrowband systems, all of these characteristics can be considered as frequency independent (i.e., constant within the considered frequency band).

In wideband systems, antenna characteristics have to be considered as frequency dependent. In UWB systems, additional characteristics must be introduced to take into account the transient radiation and to reveal phase variation effects. UWB antennas are considered as linear

Manuscript received December 21, 2006; revised April 26, 2007.

The authors are with the Polytechnic National Institute of Grenoble, LCIS, 26000 Valence, France (e-mail: Yvan.Duroc@esisar.inpg.fr).

Digital Object Identifier 10.1109/TAP.2007.908573 time invariant systems defined in the frequency domain by a complex transfer function, and in the time domain by the associated impulse response [4], [5]. The antenna characteristics also depend on the signal propagation direction. Transfer functions and impulse responses modeling UWB antennas are then spatial vectors [6]. Such a characterization provides especially the radiated and received transient waveforms of any arbitrary waveform excitation and antenna orientation. In this context, Section III-C. presents a method providing a time-frequency model from a parametric approach.

Otherwise in circuit design, antennas are considered as loaded impedances. In narrowband systems, an antenna is simply represented by a $50 \Omega$ resistor or a RLC parallel circuit to consider mismatching. However when considering UWB antennas, the circuit modeling becomes more complex as several adjacent resonances have to be taken into account [7]. Section III-D. proposes an efficient method also based on a parametric approach to obtain an equivalent circuit of antenna input impedances.

\section{MODELING CONSIDERATIONS}

\section{A. Generalities on Parametric Approaches}

Parametric approaches are often used in the modeling field because analytical and compressed expressions can be achieved from measurement. In the time domain, two of the most popular linear methods are the polynomial method and the matrix pencil method. The polynomial method was first developed by Prony in 1795 but was further developed in [8]. The matrix pencil technique [9] is more recent and more computationally efficient because the determination of the poles $z_{n}$ is a one-step process. Prony and matrix pencil methods use the same projection in a base of exponential functions. The mathematical model can be formulated as

$$
h(t)=\sum_{n=1}^{N} R_{n} e^{s_{n} t}, \quad t \geq 0
$$

with $R_{n}=G_{n} e^{j \varphi_{n}}, G_{n} \in P, \varphi_{n} \in P$ and $s_{n}=\alpha_{n}+2 \pi j f_{n}$, $\alpha_{n} \in P, f_{n} \in P . x(t)$ is the observed time response, $\left\{R_{n}\right\}$ are the residues (complex amplitudes), $\left\{s_{n}\right\}$ are the poles and $N$ is the order of the model. Moreover, $\left\{\alpha_{n}\right\}$ are the damping factors and $\left\{f_{n}\right\}$ are the frequencies.

Then, after sampling and with $z_{n}=\exp \left(s_{n} T_{e}\right)$, the sequence can be rewritten as

$$
h(k)=\sum_{n=1}^{N} R_{n} z_{n}^{k}, k \geq 0 .
$$

This model has notably the advantage of computing in an efficient way the frequency domain from the time domain by the knowledge of poles and residues as shown in the following relation:

$$
\operatorname{LT}[h(t)]=H(p)=\sum_{n=1}^{N} \frac{R_{n}}{p-s_{n}}
$$

where the operator LT corresponds to the Laplace transform.

The model order will usually not be known. There is no straightforward method to compute it. However, two solutions are generally applied: to choose the most significant residues or to use the singular value decomposition (SVD). The accuracy of the fit model can be achieved by calculating the "mean square error" of the difference between the model and the measured impulse responses (or transfer functions). In the following analysis, the signal to noise ratio (SNR) is deduced from the power of the obtained error. 